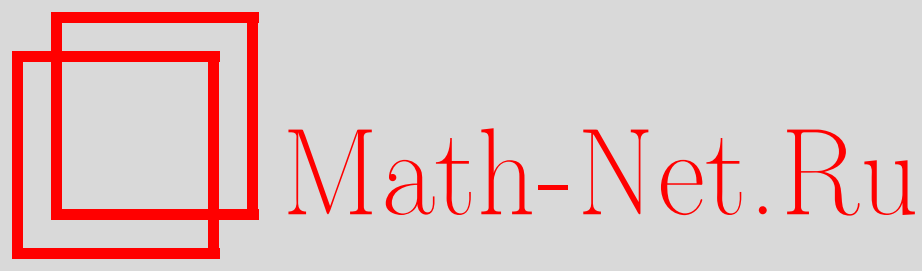

А. Ю. Аникин, Либрации и расщепление нижних уровней оператора Шредингера с потенциалом типа двойной ямы в многомерном случае, ТМ $\Phi, 2013$, том 175 , номер 2, 193-205

DOI: https://doi.org/10.4213/tmf8463

Использование Общероссийского математического портала Math-Net.Ru подразумевает, что вы прочитали и согласны с пользовательским соглашением http://www.mathnet.ru/rus/agreement

Параметры загрузки:

IP : 3.85 .5 .30

26 апреля 2023 г., 15:26:45

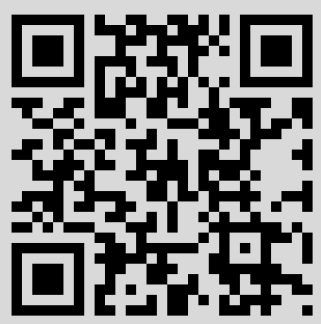




\section{ЛИБРАЦИИ И РАСЩЕПЛЕНИЕ НИЖНИХ УРОВНЕЙ ОПЕРАТОРА ШРЕДИНГЕРА С ПОТЕНЦИАЛОМ ТИПА ДВОЙНОЙ ЯМЫ В МНОГОМЕРНОМ СЛУЧАЕ}

Выведена асимптотическая формула для расщепления нижних собственных значений $n$-мерного оператора Шредингера с потенциалом с двумя симметричными ямами. В отличие от известной формулы из работы Маслова, Доброхотова и Колокольцова полученная формула имеет вид $A(h) e^{-S / h}(1+o(1))$, где $S$ - действие на периодической траектории (либрации) классической системы с перевернутым потенциалом, а не действие на двоякоасимптотической траектории. В такой записи главный член предэкспоненциального множителя принимает более элегантный вид. При выводе используется просто преобразование асимптотических формул из указанной работы без выхода за пределы классической механики.

Ключевые слова: туннельный эффект, оператор Шредингера, расщепление собственных значений, квантовая двойная яма, либрации.

DOI: $10.4213 / \operatorname{tmf} 8463$

\section{1. ВВЕДЕНИЕ}

Рассмотрим оператор Шредингера

$$
\widehat{H}=-\frac{h^{2}}{2} \Delta+V(x), \quad x \in \mathbb{R}^{n},
$$

такой, что:

Н1) $V(x) \geqslant 0, V \in C^{\infty}$ и $V(x) \rightarrow \infty$ при $x \rightarrow \infty$;

Н2) функция $V\left(x_{1}, x_{2}, \ldots, x_{n}\right)$ четная по переменной $x_{1}$;

Н3) существуют ровно два минимума $V\left(B_{1}\right)=V\left(B_{2}\right)=0$ (симметричных относительно гиперплоскости $\left.x_{1}=0\right)$, причем матрица $V_{x x}^{\prime \prime}\left(B_{i}\right)$ имеет собственные значения $\omega_{1}^{2}, \ldots, \omega_{n}^{2}$, где

$$
2 \omega_{1}<\min _{j \geqslant 2} \omega_{j}
$$

(здесь $\left.\omega_{j}>0\right)$;

* Московский государственный технический университет им. Н.Э. Баумана, Москва, Россия. E-mail: anikin83@inbox.ru 
H4) лагранжева система $L=K-U$ со стандартной кинетической энергией $K$ и "перевернутым" потенциалом $U=-V$ имеет единственную траекторию $\gamma$ (называемую инстантоном), двоякоасимптотическую к неустойчивым равновесиям $B_{i}$; при этом $\gamma$ удовлетворяет двум условиям невырожденности:

а) траектория $\gamma$ подходит к неустойчивому равновесию в неособом направлении (т. е. в направлении, отвечающем наименьшей частоте $\omega_{1}$ ),

б) асимптотические многообразия неустойчивых равновесий (в фазовом пространстве) пересекаются по $\gamma$ трансверсально.

Условие (1) носит технический характер и, вероятно, может быть снято. Оно играет роль при использовании специфических координат вблизи траектории $\gamma$.

Хорошо известно, что спектр оператора $\widehat{H}$ дискретный. Также известно (см., например, работу [1]), что с точностью $O\left(h^{\infty}\right)$ нижняя часть спектра дважды вырожденна (состоит из дважды взятого спектра любой из двух ям по отдельности). На самом деле спектр не является вырожденным из-за туннельного эффекта, и разность между соответствующими собственными значениями (называемая расщеплением) экспоненциально мала (относительно $h$ ).

Асимптотическому поведению расщепления собственного значения было посвящено множество работ. Нас будет интересовать расщепление самой нижней пары собственных значений $E_{0}, E_{1}$. Оказывается (см. работы [2]-[4] в одномерном случае и работы [5], [6] в многомерном случае), что $\lim _{h \rightarrow 0} h \ln \left(E_{1}-E_{0}\right)=-S_{0}$, где $S_{0}-$ действие Мопертюи на всем инстантоне. Напомним, что действием Мопертюи на траектории $x:[a, b] \rightarrow \mathbb{R}^{n}$ с энергией $E$ системы $L$ в конфигурационном пространстве $\mathbb{R}^{n}$ называется функционал

$$
\int_{a}^{b} \sqrt{2(E-U(x))\langle\dot{x}, \dot{x}\rangle} d t .
$$

Здесь $\langle\cdot, \cdot\rangle$ - стандартное скалярное произведение. В дальнейшем по умолчанию “действие" будет всегда означать "действие Мопертюи".

Далее, в работах [6] показано, что

$$
E_{1}-E_{0}=A(h) e^{-S_{0} / h}
$$

где $A(h) \sim \sqrt{h}$. В одномерном случае главный член $A(h)$ был рассчитан в статьях [3], [7], [8]. Там же были получены аналогичные асимптотики для расщеплений последующих пар нижних собственных значений $\left(E_{3}-E_{2}, E_{5}-E_{4}\right.$ и т. д.).

В многомерном случае к настоящему времени известен главный член $A(h)$ для самой нижней пары собственных значений [9]. В указанной работе главный член $A(h)$ выражался в терминах уравнений в вариациях вблизи инстантона. Отметим, что в работах [6], а также [10] получены весьма общие результаты (охватывающие случаи не только нижних пар собственных значений, но также таких пар собственных значений, которые стремятся к $E \neq 0$ при $h \rightarrow 0$ ). Однако такие полезные явные асимптотики для $A(h)$ в них не выписаны.

Асимптотика для $A(h)$ имеет громоздкий вид даже в одномерном случае. В одномерном случае можно сделать следующее интересное наблюдение. Формула (2) приобретает более изящный вид, если в показателе экспоненты вместо $S_{0}$ поставить 
другое действие. Пусть $S_{h}$ - половина действия на периодической траектории системы $L=K-U$ с энергией $E=-\omega h / 2$, где $\omega=\sqrt{2 V^{\prime \prime}\left(B_{i}\right)}$. Оказывается [3], [6] (см. также работу [11]), что

$$
E_{1}-E_{0}=\sqrt{\frac{\pi}{e}} \frac{\omega h}{\pi} e^{-S_{h} / h}(1+o(1)) .
$$

В этой формуле $\omega h$ имеет размерность энергии, а остальные величины являются безразмерными. Отметим, что соотношение (3) напоминает следующую формулу Ландау-Лифшица [12] (см. также статьи [13]) для расщепления верхних собственных значений. Зафиксируем $E>0$; пусть $n(h) \rightarrow \infty$ так, что $E_{n(h)}^{+}, E_{n(h)}^{-} \rightarrow E$ при $h \rightarrow 0$, тогда

$$
E_{n(h)}^{+}-E_{n(h)}^{-}=\frac{\omega_{0} h}{\pi} e^{-S / h}(1+o(1)),
$$

где $\omega_{0}$ - частота движения вдоль траектории системы $\bar{L}=K-V$ (с исходным потенциалом) с энергией $E$, а $S$ - половина действия на периодической траектории системы $L=K-U$ с энергией $-E$.

Более того, в работах [6] (а также в статьях [3]) для пар нижних собственных значений ( $n$ фиксировано, а $h \rightarrow 0$ ) получена асимптотика вида

$$
E_{2 n+1}-E_{2 n}=b_{n} \frac{\omega h}{\pi} e^{-S / h}(1+o(1))
$$

где $S$ - половина действия на периодической траектории системы $L=K-U$ с энергией $-E_{2 n}=-\omega h(1+2 n) / 2+o(h)$, а $b_{n}$ - безразмерные константы (в частности, $\left.b_{0}=\sqrt{\pi / e}\right)$. Оказалось, что $b_{n} \rightarrow 1$ при $n \rightarrow \infty$, что прекрасно согласуется с формулой (4).

В работе [14] был найден замечательный подход к обобщению равенства (3) на многомерный случай. Это обобщение не является очевидным: угадать окончательный ответ совсем не просто. Авторам работы [14] удалось сформулировать конечный результат, однако некоторые рассуждения были схематичными или не вполне строгими. Кроме того, в указанной работе рассматривался только двумерный случай. Обобщение некоторых конструкций на случай произвольного $n$ требует дополнительных усилий. В настоящей работе дается полный вывод асимптотической формулы расщепления (теорема 1) в общем многомерном случае. При этом необходимо отметить, что многие идеи мы черпаем из работы [14].

Чтобы сформулировать теорему 1, мы изучим подробнее лагранжеву систему $L=K-U$ с перевернутым потенциалом. Можно показать, что в рамках предположений $\mathrm{H} 1-\mathrm{H} 4$ система $L=K-U$ имеет однопараметрическое семейство периодических движений $\gamma_{\varepsilon}$ (параметром является энергия $E=-\varepsilon$, где $\varepsilon>0$ достаточно мало). Скорость $\gamma_{\varepsilon}$ обращается в нуль дважды на периоде. Такие движения называются либрациями (bounce orbits или brake orbits). Существование либраций - не вполне очевидный факт (доказано в работах [15], [16] в несколько ином контексте). Кроме того, ясно, что эти периодические движения являются гиперболическими (имеют $n-1$ положительных и $n-1$ отрицательных показателей Флоке).

Семейство либраций удобно параметризовать величиной $J=S / 2 \pi$, где $S$ - действие на периоде либрации. Обозначим через $H_{0}(J)$ энергию либрации с действием $S$, а через $\lambda_{j}(J), 2 \leqslant j \leqslant n,-$ положительные показатели Флоке этой либрации. 
Теорема 1. Разность двух наименъших собственных значений оператора $\widehat{H}$, удовлетворяющего условиям $\mathrm{H} 1-\mathrm{H} 4$, имеет асимптотику

$$
E_{1}-E_{0}=\sqrt{\frac{\pi}{e}} \frac{\omega_{1} h}{\pi} e^{-\bar{J}_{h} \pi / h}(1+o(1)), \quad h \rightarrow 0,
$$

где $\bar{J}_{h}$ находится из соотношения

$$
H_{0}\left(\bar{J}_{h}\right)-\frac{h}{2} \sum_{j=2}^{n} \lambda_{j}\left(\bar{J}_{h}\right)=-\left(\omega_{1}+\cdots+\omega_{n}\right) \frac{h}{2} .
$$

Чтобы проиллюстрировать эту теорему, рассмотрим случай разделения переменных. Пусть $V=V_{1}\left(x_{1}\right)+V_{2}\left(x_{2}\right)$, где $V_{1}$ - одномерная двойная яма (при этом выполнены предположения Н1-H3), а $V_{2}$ - одномерная яма в начале координат. Легко видеть, что в данном случае расщепление будет совпадать с расщеплением нижних собственных значений одномерного оператора с потенциалом $V_{1}$. Таким образом, имеет место расщепление (3). С другой стороны, $\lambda_{2}=\omega_{2}$, поэтому теорема 1 дает тот же самый результат.

Идея доказательства теоремы 1 следующая. В конфигурационном пространстве $\mathbb{R}^{n}$ вводятся естественные координаты вблизи $\gamma$ : координата $s$ вдоль инстантона $\gamma$ и "трансверсальные" координаты $q_{2}, \ldots, q_{n}$. В этих координатах мы записываем уравнение в вариациях в окрестности инстантона $\gamma$. Пусть

$$
\widehat{H}^{\mathrm{I}}=-\frac{h^{2}}{2} \frac{d^{2}}{d s^{2}}+V_{0}
$$

- одномерный оператор Шредингера, где $V_{0}$ - значение потенциала $V$ в точке инстантона с координатой $s$.

Применяя формулу расщепления вида $(2)$ (с амплитудой $A(x)$ из работы [9]) для $\widehat{H}$ и $\widehat{H}^{\mathrm{I}}$, получаем соотношение

$$
E_{1}-E_{0}=\mathcal{T}\left(E_{1}^{\mathrm{I}}-E_{0}^{\mathrm{I}}\right)(1+o(1)),
$$

где $E_{0}^{\mathrm{I}}, E_{1}^{\mathrm{I}}$ - нижние собственные значения оператора $\widehat{H}^{\mathrm{I}}$ (напомним, что для любого числа степеней свободы $A(h) \sim \sqrt{h})$.

Число $\mathcal{T}$ выражается в терминах краевой задачи для уравнения в вариациях в окрестности инстантона $\gamma$. В статье [14] показано, что в двумерном случае $\mathcal{T}$ можно интерпретировать как квадратный корень из амплитуды рассеяния.

Далее, применяя соотношение (3), получим

$$
E_{1}-E_{0}=\mathcal{T} \sqrt{\frac{\pi}{e}} \frac{\omega_{1} h}{\pi} e^{-S_{h} / h}(1+o(1)),
$$

где $S_{h}$ - действие на либрации системы $L=T-U$ с энергией $-h \omega_{1} / 2$. Здесь также используется факт близости (с погрешностью $O\left(h^{1+\epsilon}\right)$, где $\epsilon>0$ ) действий на либрациях $n$-мерной и соответствующей одномерной лагранжевых систем с равными энергиями [17].

Затем мы находим асимптотику суммы всех положительных показателей Флоке либрации с большим периодом (где возникает та же самая величина $\mathcal{T}$ ). Далее мы 
пишем асимптотику для $S_{h}-\pi \bar{J}_{h}$ и после подстановки в (6) получаем требуемое утверждение.

Таким образом, при выводе теоремы 1 используются известные результаты об энергетическом расщеплении [9]. Важно отметить, что мы не выходим при этом за рамки классической механики.

\section{2. СПРЯМЛЯЮЩИЕ КООРДИНАТЫ}

В настоящем разделе мы изучаем лагранжеву систему $L=K-U$. Введем некоторые координаты в окрестности инстантона $\gamma$. В этих координатах записывается уравнение в вариациях вблизи $\gamma$.

Пусть

$$
x=r(s), \quad-l \leqslant s \leqslant l, \quad r(-l)=B_{1}, \quad r(l)=B_{2},
$$

- натуральная параметризация инстантона $\gamma$ относительно евклидовой метрики, а $e_{j}(s), j=1, \ldots, n,-$ репер Френе. Из соображений симметрии ясно, что $y_{0}=r(0)$ лежит на гиперплоскости $x_{1}=0$. Сделаем замену координат $x \mapsto s, q_{2}, \ldots, q_{n}$ по формуле

$$
x=r(s)+\sum_{j=2}^{n} q_{j} e_{j}(s) .
$$

Пусть $k_{j}(s), j=1, \ldots, n-1,-$ кривизны траектории $\gamma\left(k_{1}-\right.$ кривизна, $k_{2}-$ кручение, взятое с обратным знаком, в случае $n=3$ ). Из гипотезы Н3 следует, что все $k_{j} \rightarrow 0$ при $s \rightarrow \pm l$. Пусть $P, Q_{j}$ - импульсы, канонически сопряженные $s, q_{j}$.

ПреДЛОЖЕНИЕ 1. Кинетическая энергия в координатах $s, q_{j}, P, Q_{j}$ имеет вид

$$
K=\frac{P^{2}}{2\left(1-q_{2} k_{1}\right)^{2}}+\sum_{j=2}^{n}\left(P Q_{j} \mu_{j}+\frac{Q_{j}^{2}}{2}\right)+O\left(q^{2}+Q^{2}\right)^{3 / 2},
$$

где $\mu_{2}=-q_{3} k_{2}$ при $n>2, \mu_{2}=0$ при $n \leqslant 2$,

$$
\mu_{j}=q_{j-1} k_{j-1}-q_{j+1} k_{j}, \quad j=3, \ldots, n-1,
$$

$u \mu_{n}=q_{n-1} k_{n-1}$.

ДокАзАТЕЛЬство. Из формул Френе следует, что

$$
\dot{r}=\dot{s}\left(1-q_{2} k_{1}\right) e_{1}+\sum_{j=2}^{n}\left(\dot{q}_{j}+\dot{s} \mu_{j}\right) e_{j} .
$$

Поэтому

$$
K=\frac{1}{2} \dot{r}^{2}=\frac{1}{2}\left[\dot{s}^{2}\left(1-q_{2} k_{1}\right)^{2}+\sum_{j=2}^{n}\left(\dot{q}_{j}+\dot{s} \mu_{j}\right)^{2}\right],
$$

следовательно,

$$
P=\dot{s}\left(\left(1-q_{2} k_{1}\right)^{2}+\sum_{j=2}^{n} \mu_{j}^{2}\right)+\sum_{j=2}^{n} \dot{q}_{j} \mu_{j}, \quad Q_{j}=\dot{q}_{j}+\dot{s} \mu_{j} .
$$

Преобразование Лежандра (выражаем производные $\dot{s}$ и $\dot{q}_{j}$ из соотношений (7) и подставляем в $\left.K-\dot{s} P-\sum_{j=2}^{n} \dot{q}_{j} Q_{j}\right)$ дает требуемое выражение для кинетической энергии в фазовом пространстве. Предложение 1 доказано. 
Пусть

$$
U=U_{0}(s)+\sum_{j=2}^{n} U_{j}(s) q_{j}+\frac{1}{2} \sum_{2 \leqslant i, j \leqslant n} U_{i j}(s) q_{i} q_{j}+O\left(q^{3}\right) .
$$

Поскольку $q_{j}=Q_{j}=0$ всюду на $\gamma$, то $U_{2}=2 k_{1} U_{0}$ и $U_{j}=0$ при $j \geqslant 3$. Таким образом, функция Гамильтона нашей системы имеет вид

$$
\begin{aligned}
H= & \frac{P^{2}}{2}\left(1+2 k_{1} q_{2}+3 k_{1}^{2} q_{2}^{2}\right)+\sum_{j=2}^{n}\left(P Q_{j} \mu_{j}+\frac{Q_{j}^{2}}{2}\right)+ \\
& +U_{0}(s)\left(1+2 k_{1} q_{2}\right)+\frac{1}{2} \sum_{2 \leqslant i, j \leqslant n} U_{i j}(s) q_{i} q_{j}+O\left(q^{2}+Q^{2}\right)^{3 / 2} .
\end{aligned}
$$

В новых координатах инстантон $\gamma$ задается в виде $s=s_{0}(t), P=P_{0}(t), q_{j}=Q_{j}=0$.

ПредлОЖенИЕ 2. Уравнение в вариациях вблизи $\gamma$ имеет вид $\dot{\xi}=A \xi$ (мь приняли следующий порядок переменных: $\left.s, P, q_{2}, \ldots, q_{n}, Q_{2}, \ldots, Q_{n}\right)$, где

$$
A=\left(\begin{array}{ccc}
\Omega_{0} & \Lambda_{1} & 0 \\
0 & R & E \\
\Lambda_{2} & \Omega & R
\end{array}\right) \in \mathbb{R}^{2 n \times 2 n}, \quad \Omega_{0}=\left(\begin{array}{cc}
0 & 1 \\
U^{\prime \prime}\left(s_{0}(t)\right) & 0
\end{array}\right) \in \mathbb{R}^{2 \times 2}
$$

Здесъ $E, \Omega, R \in \mathbb{R}^{(n-1) \times(n-1)}, E-$-диничная матрица и $\Omega=\left(\omega_{i j}\right), R=\left(r_{i j}\right)$, где

$$
\omega_{i j}=-U_{i+1, j+1}-3 \sigma_{1}^{2} \delta_{i 1} \delta_{j 1}, \quad r_{i j}=\delta_{i, j+1} \sigma_{i}-\delta_{i, j-1} \sigma_{j}, \quad i, j=1, \ldots, n-1
$$

$\left(\delta_{i j}\right.$ - символ Кронекера $)$, а $\Lambda_{1} \in \mathbb{R}^{2 \times(n-1)}, \Lambda_{2} \in \mathbb{R}^{(n-1) \times 2}$ задаются равенствами

$$
\Lambda_{1}=\left(\begin{array}{cccc}
2 \sigma_{1} & 0 & \ldots & 0 \\
-2 \sigma_{0} & 0 & \ldots & 0
\end{array}\right), \quad \Lambda_{2}=\left(\begin{array}{cccc}
-2 \sigma_{0} & 0 & \ldots & 0 \\
-2 \sigma_{1} & 0 & \ldots & 0
\end{array}\right)^{\mathrm{T}}
$$

в которых $\sigma_{0}(t)=k_{1}\left(s_{0}(t)\right) U^{\prime}\left(s_{0}(t)\right) u \sigma_{j}(t)=k_{j}\left(s_{0}(t)\right) P_{0}(t) n p u 1 \leqslant j \leqslant n-1$.

ДокАзАТЕЛЬСтво получается прямой проверкой.

Теперь в системе Гамильтона сделаем изоэнергетическую редукцию к $H=0$. Для этого выразим $P=G(s, q, Q)$ из уравнения $H=0$. Получим неавтономную систему с $n-1$ степенями свободы:

$$
\frac{d q_{j}}{d s}=\frac{H_{Q_{j}}^{\prime}}{H_{P}^{\prime}}, \quad \frac{d Q_{j}}{d s}=-\frac{H_{q_{j}}^{\prime}}{H_{P}^{\prime}} .
$$

ПрЕДЛОЖЕНИЕ 3. Уравнение в вариациях системы (8) вблизи инстантона $\gamma$ (вблизи $q=Q=0)$ после замены независимой переменной $s=s_{0}(t)$ принимает вид

$$
\frac{d \xi}{d t}=\check{A} \xi, \quad \check{A}=\left(\begin{array}{cc}
R & E \\
\Omega & R
\end{array}\right)
$$

(здесъ порядок переменных $\left.q_{2}, \ldots, q_{n}, Q_{2}, \ldots, Q_{n}\right)$. 
ДокАЗАтЕЛЬство. Легко видеть, что $H_{P}^{\prime}=P+O\left(q^{2}+Q^{2}\right)^{1 / 2}$. Пусть $G_{0}(s)=$ $G(s, 0,0)$, тогда

$$
G=G_{0}-\sum_{j=2}^{n}\left(\left.q_{j} \frac{H_{q_{j}}^{\prime}}{H_{P}^{\prime}}\right|_{\gamma}+\left.Q_{j} \frac{H_{Q_{j}}^{\prime}}{H_{P}^{\prime}}\right|_{\gamma}\right)+O\left(q^{2}+Q^{2}\right),
$$

и ясно, что $G=G_{0}+O\left(q^{2}+Q^{2}\right)$. Поэтому из (8) получаем

$$
\frac{d \xi}{d s}=\frac{1}{G_{0}} \tilde{A} \xi, \quad \tilde{A}=\left(\begin{array}{cc}
\widetilde{R} & E \\
\Omega & \widetilde{R}
\end{array}\right),
$$

где матрица $\widetilde{R}$ получается из $R$ заменой $P_{0}$ на $G_{0}(s)$ и всех $k_{j}\left(s_{0}(t)\right)$ на $k_{j}(s)$. Покажем, как выводится уравнение для $\dot{Q}_{2}$ (вывод остальных уравнений еще более простой). Запишем соответствующее уравнение системы Гамильтона:

$$
\begin{aligned}
\dot{Q}_{2} & =-P^{2} k_{1}-3 P^{2} k_{1}^{2} q_{2}-P Q_{3} k_{2}-2 U_{0} k_{1}-\sum_{j=2}^{n} U_{2 j} q_{j}+O\left(q^{2}+Q^{2}\right)= \\
& =-k_{1}\left(G_{0}^{2}+2 U_{0}\right)-3 G_{0}^{2} k_{1}^{2} q_{2}-G_{0} k_{2} Q_{3}-\sum_{j=2}^{n} U_{2 j} q_{j}+O\left(q^{2}+Q^{2}\right)= \\
& =-3 G_{0}^{2} k_{1}^{2} q_{2}-G_{0} k_{2} Q_{3}-\sum_{j=2}^{n} U_{2 j} q_{j}+O\left(q^{2}+Q^{2}\right),
\end{aligned}
$$

что дает соответствующее уравнение (10). После замены независимой переменной $s=s_{0}(t)$ система (10) приводится к виду (9).

\section{3. РАСЩЕПЛЕНИЕ В ТЕРМИНАХ УРАВНЕНИЯ В ВАРИАЦИЯХ}

Цель настоящего раздела - переписать в удобном для нас виде асимптотику расщепления нижнего энергетического уровня из работ [9]. Основным результатом является теорема 2.

Рассмотрим краевую задачу для уравнения в вариациях:

$$
\dot{\Xi}=A \Xi, \quad \Xi_{q}(0)=E, \quad \Xi_{q}(-\infty)=0 .
$$

Здесь $\Xi \in \mathbb{R}^{2 n \times n}$, а $\Xi_{q} \in \mathbb{R}^{n \times n}$ - матрица, составленная из строк матрицы $\Xi$ с номерами $1,3,4, \ldots, n, n+1$ (т. е. строк с номерами, соответствующими координатам). Аналогично $\Xi_{Q} \in \mathbb{R}^{n \times n}$ - матрица, составленная из остальных строк матрицы $\Xi$ (т. е. строк, соответствующих импульсам). Задача (11) имеет единственное решение, поскольку асимптотические многообразия положений равновесия пересекаются трансверсально с многообразиями $\left\{s=0, q_{j}=\delta_{i j}\right\}$.

Положим

$$
\beta_{n}=\lim _{t \rightarrow-\infty} e^{-\left(\omega_{1}+\cdots+\omega_{n}\right) t} \operatorname{det} \Xi_{q}(t) .
$$

Далее, пусть $\check{\Xi}_{Q} \in \mathbb{R}^{(n-1) \times(n-1)}$ получается из матрицы $\Xi_{Q}$ удалением первого столбца и первой строки. В работах [9] получено следующее уточнение формулы (2):

$$
E_{1}-E_{0}=2 \sqrt{\frac{h}{\pi}} \beta_{n}\left(\operatorname{det} \check{\Xi}_{Q}(0)\right)^{-1 / 2} \sqrt{\omega_{1} \ldots \omega_{n}}\left|\dot{s}_{0}(0)\right| e^{-S_{0} / h}(1+o(1)) .
$$


Рассмотрим, как и выше, одномерный оператор (5), $V_{0}=-U_{0}$, удовлетворяющий условиям Н1-Н3. Из соотношения (12) следует, что расщепление нижних собственных значений оператора $\widehat{H}^{\mathrm{I}}$ описывается формулой

$$
E_{1}^{\mathrm{I}}-E_{0}^{\mathrm{I}}=2 \sqrt{\frac{h}{\pi}} \beta_{1} \sqrt{\omega_{1}}\left|\dot{s}_{0}(0)\right| e^{-S_{0} / h}(1+o(1)),
$$

откуда

$$
E_{1}-E_{0}=\mathcal{T}\left(E_{1}^{\mathrm{I}}-E_{0}^{\mathrm{I}}\right), \quad \mathcal{T}=\beta_{n} \beta_{1}^{-1}\left(\operatorname{det} \check{\Xi}_{Q}(0)\right)^{-1 / 2} \sqrt{\omega_{2} \ldots \omega_{n}} .
$$

Рассмотрим краевую задачу, аналогичную (11), но теперь для уравнения в вариациях (9):

$$
\dot{\Theta}=\check{A} \Theta, \quad \Theta_{q}(0)=E, \quad \Theta_{q}(-\infty)=0,
$$

где $\Theta \in \mathbb{R}^{2(n-1) \times(n-1)}$, матрицы $\Theta_{q}, \Theta_{Q} \in \mathbb{R}^{(n-1) \times(n-1)}$, матрица $\Theta_{q}$ состоит из первых $n-1$ строк матрицы $\Theta$, а $\Theta_{Q}$ - из остальных строк матрицы $\Theta$.

Теорема 2. Пусть $\Theta$-решение задачи (14), положим

$$
a_{n}=\operatorname{det} \Theta_{Q}(0), \quad \check{\beta}_{n}=\lim _{t \rightarrow-\infty} e^{-\left(\omega_{2}+\cdots+\omega_{n}\right) t} \operatorname{det} \Theta_{q}(t) .
$$

Тогда имеет место асимптотическое равенство (6), причем

$$
\mathcal{T}=\check{\beta}_{n} a_{n}^{-1 / 2} \sqrt{\omega_{2} \ldots \omega_{n}} .
$$

ДокАЗАТЕЛЬСтво. Обозначим через $S_{h}^{\mathrm{I}}$ половину действия на либрации одномерной системы $L=K-U_{0}$ с энергией $-\omega h / 2$. В работе [17] было доказано, что $S_{h}-S_{h}^{\mathrm{I}}=O\left(h^{1+\epsilon}\right)$ для некоторого $\varepsilon>0$ (эта оценка была получена для $n=2$, однако все рассуждения легко могут быть перенесены на случай произвольного $n$ ). После подстановки соотношения (3) в (13) получаем формулу (6).

Нам остается выразить $\mathcal{T}$ в терминах задачи (14).

ПреДЛОЖЕНИЕ 4. Пусть $\Theta=\left.\left(\theta_{i j}\right)\right|_{i=3 ; j=2} ^{2 n ; n}-$ решение задачи (14). Тогда матрииа $\Xi=\left.\left(\xi_{i j}\right)\right|_{i=1 ; j=1} ^{2 n ; n}$ такая, что

$$
\begin{aligned}
& \xi_{i j}=\theta_{i j}, \quad i=3, \ldots, 2 n, \quad j=2, \ldots, n, \\
& \xi_{i 1}=0, \quad i=2, \ldots, n, \\
& \xi_{11}=\frac{\dot{s}_{0}(t)}{\dot{s}_{0}(0)}, \quad \xi_{21}=\frac{\ddot{s}_{0}(t)}{\dot{s}_{0}(0)}, \quad \xi_{1 j}=\dot{s}_{0}(t) \varphi_{j}(t), \quad \xi_{2 j}=-U_{0}^{\prime}\left(\dot{s}_{0}(t)\right) \varphi_{j}(t), \\
& \varphi_{j}(t)=2 \int_{0}^{t} k_{1}\left(s_{0}(t)\right) \theta_{3 j}(t) d t, \quad j=2, \ldots, n,
\end{aligned}
$$

является решением задачи (11).

ДокАзАтЕЛьство. Уравнения задачи (11) можно записать в виде

$$
\begin{gathered}
\dot{\xi}_{11}=\xi_{21}, \quad \dot{\xi}_{21}=-U_{0}^{\prime \prime} \xi_{11}, \quad \xi_{11} U_{0}^{\prime}+\xi_{21} P_{0}=0, \\
\dot{\xi}_{1 j}=\xi_{2 j}+2 k_{1} P_{0} \theta_{3 j}, \quad \dot{\xi}_{2 j}=-U_{0}^{\prime \prime} \xi_{1 j}-2 k_{1} P_{0} \theta_{3 j}, \quad \xi_{1 j} U_{0}^{\prime}+\xi_{2 j} P_{0}=0
\end{gathered}
$$


при $j \geqslant 2$; здесь $U_{0}^{\prime}=U_{0}^{\prime}\left(s_{0}(t)\right), U_{0}^{\prime \prime}=U_{0}^{\prime \prime}\left(s_{0}(t)\right), P_{0}=P_{0}(t)$. Оставшиеся уравнения удовлетворяются автоматически, поскольку $\Theta$ - решение задачи (14).

Ясно, что функции $\xi_{11}, \xi_{21}$ из (17) удовлетворяют уравнениям (19) и граничным условиям. Найдем функцию $\xi_{1 j}=\dot{s}_{0}(t) \varphi_{j}(t)$, удовлетворяющую уравнениям (19) и граничным условиям. Тогда из последнего уравнения в $(20)$ имеем $\xi_{2 j}=-U_{0}^{\prime} \varphi_{j}(t)$. После подстановки $\xi_{1 j}, \xi_{2 j}$ каждое из первых двух уравнений в $(20)$ примет вид $\dot{\varphi}_{j}=2 k_{1} \theta_{3 j}$. Чтобы удовлетворить граничным условиям, возьмем $\varphi_{j}$ в виде (18). Предложение 4 доказано.

Итак, используя предложение 4, строим матрицу $\Xi$, удовлетворяющую уравнению (11). Поскольку

$$
\operatorname{det} \Xi_{q}(t)=\frac{\dot{s}_{0}(t)}{\dot{s}_{0}(0)} \operatorname{det} \Theta_{q}(t)
$$

мы имеем $\check{\beta}_{n}=\beta_{n} \beta_{1}^{-1}$. Наконец, из того что $\check{\Xi}_{Q}=\Theta_{Q}$, получаем равенство (15). Теорема 2 доказана.

\section{4. АСИМПТОТИКА СУММЫ ПОКАЗАТЕЛЕЙ ФЛОКЕ}

В настоящем разделе доказывается

Теорема 3. Пусть $\lambda_{j}(t)>0, j=2, \ldots, n,-$ показатели Флоке либрации с периодом 4t. Тогда

$$
\sum_{j=2}^{n} \lambda_{j}(t)=\sum_{j=2}^{n} \omega_{j}-\frac{\ln \mathcal{T}}{t}+o\left(\frac{1}{t}\right), \quad t \rightarrow+\infty
$$

Пусть $W_{ \pm}^{1}$ (соответственно $W_{ \pm}^{2}$ ) - устойчивое (неустойчивое) асимптотическое многообразие равновесия $B_{1}$ (соответственно $B_{2}$ ). Обозначим через $\left\langle u_{1}, \ldots, u_{k}\right\rangle$ или, проще, через $\langle u\rangle_{k}$ объем $k$-мерного параллелепипеда, построенного на векторах $u_{1}, \ldots, u_{k}$.

ПреДЛОЖЕНИЕ 5. Пусть $\Sigma_{0}-(n-1)$-мерная поверхность, лежащая в $W_{-}^{2} u$

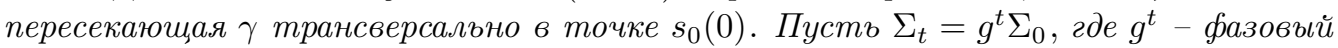
поток. Пусть $v_{1}, \ldots, v_{n-1} \in T_{s_{0}(0)} \Sigma_{0} u u_{j}=\left.d g^{t}\right|_{s_{0}(0)} v_{j}$. Тогда

$$
\left\langle u_{1}, \ldots, u_{n-1}\right\rangle=e^{\left(\lambda_{2}+\cdots+\lambda_{n}\right) t}\left\langle v_{1}, \ldots, v_{n-1}\right\rangle(1+o(1)), \quad t \rightarrow \infty .
$$

ЗАмЕчАНИЕ 1. Здесь и ниже для упрощения обозначений мы пишем $T_{s_{0}(t)} \Sigma_{0}$, где под $s_{0}(t)$ понимается точка фазового пространства $s=s_{0}(t), P=P_{0}(t), q_{j}=Q_{j}=0$.

ДокАЗАТЕЛЬСТво предложения 5 следует из того, что либрация с большим периодом близка к $\gamma$.

ПРЕДЛОЖЕНИЕ 6. Справедливо равенство

$$
\check{A}(-t)=\left(\begin{array}{cc}
R(-t) & E \\
\Omega(t) & R(-t)
\end{array}\right) .
$$

СлеДСТвиЕ 1. Пусть $\Theta(t)$ - решение уравнения (9), тогда матрица $\widetilde{\Theta}=\left(\begin{array}{c}\widetilde{\Theta}_{q} \\ \widetilde{\Theta}_{Q}\end{array}\right)$, где $\widetilde{\Theta}_{q}(t)=\Theta_{q}(-t)$ и $\widetilde{\Theta}_{Q}(t)=-\Theta_{Q}(-t)$, также является решением уравнения (9). 
ДокАЗАТЕЛЬство. Действительно, $R(t)=-R(-t)$, поскольку $k_{j}(s)=-k_{j}(-s)$ при $j \geqslant 2$. Дальнейшее доказательство проводится прямой подстановкой.

Ясно, что столбцы матрицы $[\Theta \widetilde{\Theta}]$ являются линейно независимыми решениями уравнения $(9)$. Вронскиан $\mathcal{W}(t)=\operatorname{det}[\Theta \widetilde{\Theta}]$ не зависит от $t$, следовательно, он равен $\mathcal{W}(0)=2^{n-1} a_{n}$.

Поскольку $\Theta_{q}(-\infty)=0$ и $\widetilde{\Theta}_{q}(+\infty)=0$, столбцы матрицы $\Theta(0)$ являются координатами векторов $v_{1}, \ldots, v_{n-1} \in T_{s_{0}(0)} W_{-}^{1}$, а столбцы матрицы $\widetilde{\Theta}(0)$ - координатами векторов $u_{1}, \ldots, u_{n-1} \in T_{s_{0}(0)} W_{+}^{2}$. Аналогично столбцы матрицы $\Theta(-t)$ (матрицы $\widetilde{\Theta}(-t))$ суть координаты векторов $d g^{-t} v_{j} \in T_{s_{0}(-t)} W_{-}^{1}$ (соответственно векторов $\left.d g^{-t} u_{j} \in T_{s_{0}(-t)} W_{+}^{2}\right)$. Таким образом, $\mathcal{W}(-t)$ есть объем $2(n-1)$-мерного параллелепипеда, построенного на векторах $d g^{-t} v_{j}, d g^{-t} u_{j}$.

Пусть $\pi$ - каноническая проекция из фазового пространства в конфигурационное.

ПреДЛОЖеНИЕ 7. Следующие асимптотики имеют место при $t \rightarrow+\infty$ :

$$
\begin{gathered}
\left\langle d g^{-t} v\right\rangle_{n-1}=\left\langle\pi d g^{-t} v\right\rangle_{n-1} \prod_{j=2}^{n} \sqrt{1+\omega_{j}^{2}}(1+o(1)) \\
\left\langle d g^{-t} v, d g^{-t} u\right\rangle_{2(n-1)}=\left\langle d g^{-t} v\right\rangle_{n-1}\left\langle d g^{-t} u\right\rangle_{n-1} \prod_{j=2}^{n} \frac{2 \omega_{j}}{1+\omega_{j}^{2}}(1+o(1)) .
\end{gathered}
$$

ЗАмЕчАниЕ 2. Это предложение представляет собой упражнение по многомерной аналитической геометрии. Основная идея состоит в том, что в окрестности точек $B_{i}$ легко вычислить наклон асимптотических многообразий к конфигурационному пространству. Например, в одномерном случае угол между сепаратрисой и конфигурационным пространством равен $\operatorname{arctg} \omega$.

ДоказАтельство. Докажем равенство (21). Линеаризуем систему вблизи $B_{1}$ :

$$
H_{2}=\frac{P^{2}}{2}-\frac{\omega_{1}^{2}(s+l)^{2}}{2}+\sum_{j=2}^{n} \frac{Q_{j}^{2}}{2}+\frac{1}{2} \sum_{i, j=2}^{n} U_{i j} q_{i} q_{j}
$$

Всегда можно считать, что

$$
H_{2}=\frac{P^{2}}{2}-\frac{\omega_{1}^{2} s^{2}}{2}+\sum_{j=2}^{n}\left(\frac{Q_{j}^{2}}{2}-\frac{\omega_{j}^{2} q_{j}^{2}}{2}\right),
$$

этого можно достичь с помощью поворота и сдвига конфигурационного пространства. Асимптотические многообразия линеаризованной системы - это две плоскости

$$
\Pi_{ \pm}^{1}=\left\{P= \pm \omega_{1} s ; Q_{j}= \pm \omega_{j} q_{j}\right\}
$$

Ясно, что $T_{s_{0}(-t)} W_{-}^{1} \approx \Pi_{-}^{1}$ для $t \gg 1$. Поэтому векторы $d g^{-t} v_{j}$ стремятся быть параллельными плоскости $\left\{Q_{j}=-\omega_{j} q_{j}\right\}$ (поскольку линейная оболочка векторов $d g^{-t} v_{j}$ трансверсальна инстантону). Объем $(n-1)$-мерного параллелепипеда, построенного на векторах $d g^{-t} v_{j}$, после проектирования на конфигурационное многообразие $P=Q_{j}=0$ умножается на $\left(\prod_{j=2}^{n} \sqrt{1+\omega_{j}^{2}}\right)^{-1}$. Отсюда получаем (21). 
Докажем равенство (22). Из условия Н4б следует, что $T_{s_{0}(-t)} W_{+}^{2}$ трансверсальна многообразию $W_{-}^{1}$. В соответствии с $\lambda$-леммой $T_{s_{0}(-t)} W_{+}^{2}$ стремится быть параллельной $T_{s_{0}(t)} W_{+}^{1}$ при $t \rightarrow+\infty$. Поэтому векторы $d g^{-t} u_{j}$ стремятся лечь на плоскость $\left\{Q_{j}=\omega_{j} q_{j}\right\}$ (мы вновь используем, что линейная оболочка трансверсальна инстантону). Отсюда легко получается формула (22).

ДоКАЗАТЕЛЬСТво ТЕОРЕМЫ 3. По предложению 5 при $t \rightarrow+\infty$

$$
\left\langle d g^{-t} v\right\rangle_{n-1}=\langle v\rangle_{n-1} e^{-t \lambda}(1+o(1)), \quad\left\langle d g^{-t} u\right\rangle_{n-1}=\langle u\rangle_{n-1} e^{t \lambda}(1+o(1)),
$$

где $\lambda=\lambda_{2}+\cdots+\lambda_{n}$. Поскольку $\langle v\rangle_{n-1}=\langle u\rangle_{n-1}$, по предложению 7 мы имеем

$$
\begin{aligned}
\mathcal{W}(-t) & =\left\langle d g^{-t} v, d g^{-t} u\right\rangle_{2(n-1)}=\left\langle d g^{-t} v\right\rangle_{n-1}^{2} e^{2 t \lambda} \prod_{j=2}^{n} \frac{2 \omega_{j}}{1+\omega_{j}^{2}}(1+o(1))= \\
& =\left\langle\pi d g^{-t} v\right\rangle_{n-1}^{2} e^{2 t \lambda} 2^{n-1} \omega(1+o(1))=\left(\operatorname{det} \Theta_{q}(-t) e^{t \lambda}\right)^{2} 2^{n-1} \omega(1+o(1)),
\end{aligned}
$$

где $\omega=\omega_{2} \ldots \omega_{n}$. Сравнивая с $\mathcal{W}(0)=2^{n-1} a_{n}$, видим, что

$$
\sqrt{a_{n}}=\operatorname{det} \Theta_{q}(-t) e^{t \lambda} \sqrt{\omega}(1+o(1))
$$

или

$$
e^{t \lambda}=\exp \left(t \sum_{j=2}^{n} \omega_{j}\right) \mathcal{T}^{-1}(1+o(1)) .
$$

Отсюда следует теорема 3.

\section{5. ДОКАЗАТЕЛЬСТВО ТЕОРЕМЫ 1}

ПРЕДЛОЖЕНИЕ 8. Имеет место равенство

$$
H_{0}^{\prime}(J)=\frac{2 \pi}{T(J)} .
$$

ЗАмЕчАниЕ 3. Мы доказываем это предложение, чтобы избежать обсуждения нормальной формы гамильтоновой системы в окрестности семейства периодических решений (см. работу [18]).

ДокАЗАтЕЛьство. Достаточно проверить следующий факт. Пусть $x_{\varepsilon}(t)-$ семейство периодических траекторий с энергией $\varepsilon$, действием $S(\varepsilon)$ и периодом $T_{\varepsilon}$ (где $\left.|\varepsilon|<\varepsilon_{0}\right)$, тогда

$$
\left.\frac{d S}{d \varepsilon}\right|_{\varepsilon=0}=T_{0} .
$$

Пусть $x_{\varepsilon}(t)=x_{0}(t)+\varepsilon y(t)+O\left(\varepsilon^{2}\right)$ и $T_{\varepsilon}=T_{0}+\varepsilon \tau+O\left(\varepsilon^{2}\right)$. Из $x_{0}(0)=x_{0}\left(T_{0}\right)$ и $x_{\varepsilon}(0)=x_{\varepsilon}\left(T_{\varepsilon}\right)$ следует, что $\tau \dot{x}_{0}\left(T_{0}\right)=-\left.y(t)\right|_{0} ^{T_{0}}$.

Обозначая через $S^{\mathcal{H}}(\varepsilon)=\int_{0}^{T_{\varepsilon}} L\left(\dot{x}_{\varepsilon}, x_{\varepsilon}\right) d t$ действие по Гамильтону на либрации с энергией $\varepsilon$, видим, что

$$
S^{\mathcal{H}}(\varepsilon)=S^{\mathcal{H}}(0)+\left.\varepsilon \tau\left\langle\dot{x}_{0}, \dot{x}_{0}\right\rangle\right|_{t=T_{0}}+\varepsilon \int_{0}^{T_{0}}\left(\left\langle\dot{x}_{0}, d y\right\rangle+\left\langle y, d \dot{x}_{0}\right\rangle\right)+O\left(\varepsilon^{2}\right) .
$$

Отсюда $S^{\mathcal{H}}(\varepsilon)=S^{\mathcal{H}}(0)+O\left(\varepsilon^{2}\right)$. В силу соотношения $S(\varepsilon)-S^{\mathcal{H}}(\varepsilon)=\varepsilon T_{\varepsilon}$ мы имеем $S(\varepsilon)-S_{0}=\varepsilon T_{0}+O\left(\varepsilon^{2}\right)$. Предложение 8 доказано. 
Теперь легко получить доказательство теоремы 1. Действительно, из теоремы 2 следует, что

$$
E_{1}-E_{0}=\sqrt{\frac{\pi}{e} \frac{h \omega_{1}}{\pi}} \mathcal{T} e^{-J_{h} \pi / h}(1+o(1)), \quad H_{0}\left(J_{h}\right)=-\frac{h \omega_{1}}{2},
$$

а из теоремы 3 следует, что

$$
H_{0}\left(\bar{J}_{h}\right)-\frac{h}{2}\left(\sum_{j=2}^{n} \omega_{j}-4 \frac{\ln \mathcal{T}}{T\left(\bar{J}_{h}\right)}\right)=-\frac{h}{2} \sum_{j=1}^{n} \omega_{j}+O\left(h^{1+\epsilon}\right) .
$$

По формуле Тейлора

$$
H_{0}\left(\bar{J}_{h}\right)=H_{0}\left(J_{h}\right)+\frac{2 \pi}{T\left(J_{h}\right)}\left(\bar{J}_{h}-J_{h}\right)+O\left(h^{1+\epsilon}\right),
$$

поэтому

$$
\frac{2 \pi}{T\left(J_{h}\right)}\left(\bar{J}_{h}-J_{h}\right)+\frac{2 h \ln \mathcal{T}}{T\left(J_{h}\right)}=O\left(h^{1+\epsilon}\right)
$$

или $J_{h}=\bar{J}_{h}+h \ln \mathcal{T} / \pi+O\left(h^{1+\epsilon / 2}\right)$. Подставляя последнюю формулу в $(23)$, получаем утверждение теоремы 1.

Благодарности. Автор выражает благодарность С. Ю. Доброхотову, Д. В. Трещеву и С. В. Болотину за полезные дискуссии. Работа выполнена при поддержке РФФИ (грант № 12-01-00441) и Программы поддержки ведущих научных школ (грант НШ-2519.2012.1).

\section{Список литературы}

[1] B. Simon, Ann. Inst. H. Poincaré Sect. A (N.S.), 38:3 (1983), 295-308.

[2] A. M. Polyakov, Nucl. Phys. B, 120:3 (1977), 429-458; E. Gildener, A. Patrascioiu, Phys. Rev. D, 16:2 (1977), 423-430; Erratum, 16:2 (1977), 3616-3616; S. Coleman, "The uses of instantons", Proceedings International School of Physics (Erice, Sicily, 1977), ed. A. Zichichi, Plenum, New York, 1979, 805-916.

[3] E. Harrell, Commun. Math. Phys., 60:1 (1978), 73-95; 75:3 (1980), 239-261.

[4] G. Jona-Lasinio, F. Martinelli, E. Scoppola, Commun. Math. Phys., 80:2 (1981), 223-254; J. M. Combes, P. Duclos, R. Seiler, J. Funct. Anal., 52:2 (1983), 257-301.

[5] В. П. Маслов, Тр. МИАН, 163 (1984), 150-180; В. Simon, Ann. Math. (2), 120:1 (1984), 89-118.

[6] B. Helffer, J. Sjöstrand, Commun. Part. Differ. Equ., 9:4 (1984), 337-408; Ann. Inst. H. Poincaré Phys. Théor., 42:2 (1985), 127-212.

[7] С. Ю. Славянов, Дифферени. уравнения, 5:2 (1969), 313-325.

[8] Т. Ф. Панкратова, Докл. АН СССР, 276:4 (1984), 795-798.

[9] С. Ю. Доброхотов, В.Н. Колокольцов, В.П. Маслов, ТМФ, 87:3 (1991), 323-375; С. Ю. Доброхотов, В. Н. Колокольцов, ТМФ, 94:3 (1993), 426-434.

[10] A. Martinez, J. Math. Pures App. (9), 66:2 (1987), 195-215; Bull. Soc. Math. France, 116:2 (1988), 199-229.

[11] С. А. Альбеверио, С. Ю. Доброхотов, Е. С. Семенов, ТМФ, 138:1 (2004), 116-126.

[12] Л. Д. Ландау, Е. М. Лифшиц, Теоретическая физика, т. 3: Квантовая механика. Нерелятивистская теория, Наука, М., 1974. 
[13] М.В. Федорюк, Матем. сб., 68(110):1 (1965), 81-110; А. Г. Аленицын, Дифберени. уравнения, 18:11 (1982), 1971-1975.

[14] J. Brüning, S. Yu. Dobrokhotov, E. S. Semenov, Regul. Chaotic Dyn., 11:2 (2006), 167-180.

[15] В. И. Арнольд, В. В. Козлов, А. И. Нейштадт, Математические аспекты классической и небесной механики, Эдиториал УРСС, М., 2002.

[16] С. В. Болотин, Вестн. Моск. ун-та. Сер. 1. Матем., мех., 6 (1978), 72-77.

[17] A. Yu. Anikin, Russ. J. Math. Phys., 20:1 (2013), 1-10.

[18] А. Д. Брюно, УМН, 44:2(266) (1989), 49-78.

Поступила в редакцию 24.12.2012 